\title{
Ambroxol as a novel disease-modifying treatment for Parkinson's disease dementia: protocol for a single-centre, randomized, double-blind, placebo-controlled trial
}

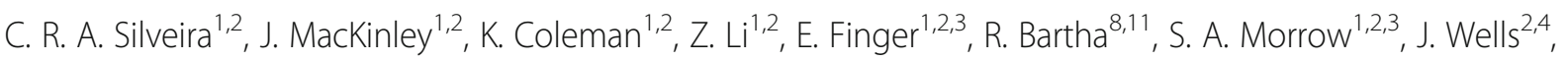
M. Borrie ${ }^{2,4}$, R. G. Tirona ${ }^{2,7}$, C. A. Rupar ${ }^{2,5}$, G. Zou ${ }^{9,11}$, R. A. Hegele 2,,11 , D. Mahuran ${ }^{10}$, P. MacDonald ${ }^{3}$, M. E. Jenkins ${ }^{2,3}$, M. Jog ${ }^{2,3}$ and S. H. Pasternak $1,2,3,7,11^{*}$ (i)

\begin{abstract}
Background: Currently there are no disease-modifying treatments for Parkinson's disease dementia (PDD), a condition linked to aggregation of the protein a-synuclein in subcortical and cortical brain areas. One of the leading genetic risk factors for Parkinson's disease is being a carrier in the gene for $\beta$-Glucocerebrosidase (GCase; gene name GBA1). Studies in cell culture and animal models have shown that raising the levels of GCase can decrease levels of a-synuclein. Ambroxol is a pharmacological chaperone for GCase and is able to raise the levels of GCase and could therefore be a disease-modifying treatment for PDD. The aims of this trial are to determine if Ambroxol is safe and well-tolerated by individuals with PDD and if Ambroxol affects cognitive, biochemical, and neuroimaging measures.

Methods: This is a phase II, single-centre, double-blind, randomized placebo-controlled trial involving 75 individuals with mild to moderate PDD. Participants will be randomized into Ambroxol high-dose ( $1050 \mathrm{mg} / \mathrm{day})$, low-dose (525 mg/day), or placebo treatment arms. Assessments will be undertaken at baseline, 6-months, and 12-months follow up times. Primary outcome measures will be the Alzheimer's disease Assessment Scale-cognitive subscale (ADAS-Cog) and the ADCS Clinician's Global Impression of Change (CGIC). Secondary measures will include the Parkinson's disease Cognitive Rating Scale, Clinical Dementia Rating, Trail Making Test, Stroop Test, Unified Parkinson's disease Rating Scale, Purdue Pegboard, Timed Up and Go, and gait kinematics. Markers of neurodegeneration will include MRI and CSF measures. Pharmacokinetics and pharmacodynamics of Ambroxol will be examined through plasma levels during dose titration phase and evaluation of GCase activity in lymphocytes.
\end{abstract}

Discussion: If found effective and safe, Ambroxol will be one of the first disease-modifying treatments for PDD.

Trial registration: ClinicalTrials.gov NCT02914366, 26 Sep 2016/retrospectively registered.

Keywords: Parkinson's disease dementia, a-Synuclein, Glucocerebrosidase, Ambroxol, Cognition

\footnotetext{
* Correspondence: spasternak@robarts.ca

${ }^{1}$ Cognitive Neurology and Alzheimer's Disease Research Centre, Parkwood

Institute - Main Building, Room A230, 550, Wellington Road, London, Ontario

N6G 0A7, Canada

${ }^{2}$ Lawson Health Research Institute, London, Ontario, Canada

Full list of author information is available at the end of the article
}

(c) The Author(s). 2019 Open Access This article is distributed under the terms of the Creative Commons Attribution 4.0 International License (http://creativecommons.org/licenses/by/4.0/), which permits unrestricted use, distribution, and reproduction in any medium, provided you give appropriate credit to the original author(s) and the source, provide a link to the Creative Commons license, and indicate if changes were made. The Creative Commons Public Domain Dedication waiver (http://creativecommons.org/publicdomain/zero/1.0/) applies to the data made available in this article, unless otherwise stated. 


\section{Background}

Parkinson's disease dementia (PDD) is a highly prevalent evolution of Parkinson's disease [1], believed to result from the aggregation of $\alpha$-synuclein in subcortical and cortical neurons $[2,3]$. Longitudinal studies have shown that by the 10-year follow up from diagnosis, $46 \%$ of individuals with Parkinson's disease had progressed to PDD [4]. Importantly, decline in cognitive function contributes to decreased quality of life [5], increased risk of institutionalization [6] and mortality [7] in individuals with Parkinson's disease. Therefore, it is not surprising that the development of new therapies to treat cognitive decline has been rated by individuals with Parkinson's disease and caregivers as one of the top 10 priorities in Parkinson's disease research [8].

Currently, the treatment for cognition in PDD includes the use of cholinesterase inhibitors in addition to dopaminergic medication [9]. Although somewhat effective in their role of alleviating symptoms, these therapies do not affect the underlying mechanisms leading to progression of the disease and patients get worse despite treatment. Thus, the development of disease-modifying treatments for PDD is critical.

A potential target for disease-modifying treatment is the enzyme $\beta$-Glucocerebrosidase (GCase; gene name GBA1). GCase is a degradative enzyme that resides in the lysosome and cleaves a neutral glycolipid, glucocerebrosidase, present in the membranes of most cells. Having mutations in both alleles of GBA1 results in Gaucher disease, which can manifest with hepatic, splenic, hematological and bone abnormalities (Type 1) or with a devastating neurological deterioration that can be rapid or chronic (Type 2 and 3, respectively). However, being a carrier for a pathological mutation in GBA1 is one of the strongest genetic risk factors for Parkinson's disease, PDD and Lewy Body dementia. Parkinson's disease patients bearing GBA1 mutation, tend to have earlier onset, a greater prevalence of cognitive decline as well as more severe cognitive impairment compared to those without the mutation [10]. In addition, reductions in GCase activity may play a role even in sporadic Parkinson's disease, as these individuals have low levels of GCase in the brain and cerebrospinal fluid [11-13].

Laboratory studies have demonstrated a direct link between GCase activity and $\alpha$-synuclein accumulation. In cultured cells, loss of GCase results in $\alpha$-synuclein accumulation and this process feeds back upon itself, with overexpression of $\alpha$-synuclein further inhibiting GCase function, and increasing GCase expression reducing $\alpha$-synuclein [14]. Moreover, reducing GCase genetically $[15,16]$ or pharmacologically [17] in animal studies results in increased $\alpha$-synuclein aggregates. Remarkably, overexpressing GCase in the brain of a Parkinson's disease mouse model reduces $\alpha$-synuclein and improves cognition $[15,18]$. Taken together, these findings suggest that increasing GCase levels could be a therapy that addresses the underlying pathophysiology of PDD to modify the course of disease progression.

Ambroxol is an expectorant that has been available for adults and children over the counter in more than 50 countries for over 30 years [19]. Ambroxol was identified by the Mahuran Lab in a High Throughput Screen as a pharmacological chaperone of GCase, an agent that stabilizes and increases the levels of GCase [20]. Pharmacological chaperones are small molecules that bind to proteins to stabilize them, increasing the number of protein molecules that fold and function correctly, and thereby reduce the amount of protein degraded. They have been proposed as therapies for a wide range of inborn errors of metabolism, a large fraction of which are caused by mutations which destabilize protein folding, leading to degradation [21-23].

In addition, Ambroxol has good lipophilicity (cLogP = 2.8) and low polar surface area (PSA $58 \AA^{2}$ ), predicting good CNS penetration. Ambroxol has an excellent safety record and has been studied in $>15,000$ patients in more than 100 trials. While the normal expectorant dose in adults is in the range of $75-100 \mathrm{mg} /$ day, doses of 1000 $\mathrm{mg} I V$ are used in pregnant women experiencing premature delivery to aid fetal lung maturation, and doses of $30 \mathrm{mg} / \mathrm{kg}$ in neonates for fetal respiratory distress syndrome [19].

Although Ambroxol was originally identified as a drug which stabilizes GCase bearing disease-causing mutations which increase its degradation, it is also able to increase the levels of normal GCase [20, 24]. As such, it can markedly increase GCase protein and activity in both normal and Gaucher disease fibroblasts (bearing GCase mutations) at concentrations in the micromolar range [20, 24]. Ambroxol treatment has also been shown to improve lysosomal biochemistry overall by indirect activation of a lysosomal master regulatory gene/transcription factor called TFEB and improved clearance of $\alpha$-synuclein [25]. More recently, Ambroxol was found to raise GCase levels and lower $\alpha$-synuclein in induced pluripotent stem cell (iPSC)-derived dopaminergic neurons from GBA1 mutation patients [26].

In mice, oral Ambroxol for 12 days increased GCase activity in the brainstem, midbrain, cortex, and striatum of wild-type as well as transgenic mice carrying a L444P mutation (i.e. a GBA1 mutation) [27]. The same study found that transgenic mice overexpressing human $\alpha$-synuclein treated with Ambroxol had a reduction in $\alpha$-synuclein levels in the brainstem and striatum compared to untreated mice. Furthermore, Ambroxol reduced S129 phosphorylation of $\alpha$-synuclein (suggested to play a critical role in $\alpha$-synuclein aggregation and formation of Lewy bodies and neuritis) in the brainstem by 
$41 \%$ in treated mice compared to untreated mice. Ambroxol has also recently been demonstrated to increase GCase levels in non-human primates [28]. Together, these results support the potential for using Ambroxol as a disease-modifying treatment for synucleinopathies such as PDD.

In pilot studies in humans, Ambroxol was effective at improving GCase function [29, 30]. In a trial aimed at Type 1 (non-neurological) Gaucher disease, 12 patients received $150 \mathrm{mg} /$ day for 6 months, and all but one had some measurable improvement. The best response was in the lightest patient (who received $3 \mathrm{mg} / \mathrm{kg} /$ day), suggesting that Ambroxol was under dosed in this study. Ambroxol has also been administered to five Japanese Gaucher disease patients with severe neurological disease, at $25 \mathrm{mg} / \mathrm{kg} /$ day or a maximum dose of $1300 \mathrm{mg} /$ day for 6-48 months [29]. Despite their advanced disease, patients had improvements in neurological symptoms (decreased myoclonus and seizure frequency). Improvements in myoclonus allowed two patients to stand steadily, control their balance, and walk again. To our knowledge, no study has tested the effects of Ambroxol on people with Parkinson's disease or PDD.

The main objectives of the present randomized controlled trial are 1) to demonstrate the safety and tolerability of Ambroxol in patients with PDD, 2) to examine the effects of Ambroxol on cognitive, biochemical, and neuroimaging measures, and 3) to acquire additional pharmacokinetic and pharmacodynamics data for use in future trials. It is hypothesized that Ambroxol will be safe and well tolerated in PDD, and may improve the course of cognitive impairment or motor function, or biomarkers of neurodegeneration in PDD by raising GCase levels in blood and CSF.

\section{Methods}

The study has been approved by Western University Health Science Research Ethics Board (Protocol ID: 105234) and Health Canada (Protocol ID: HC624-c181033) in November 2015. The trial has been registered on ClinicalTrials.gov (ID: NCT02914366).

\section{Design}

This is a phase II, single-centre, double-blind, placebo-controlled randomized clinical trial (RCT) using a parallel arm design with a 6-month open label extension period (Fig. 1).

\section{Participants}

Participants must fulfill the following inclusion criteria: 1) age greater than 50 years old; 2) Parkinson's disease (Hoehn \& Yahr stage 2-3.5) clearly established for more than 1 year before the onset of dementia; 3 ) mild to moderate dementia (defined by an upper cut off of a
Montreal Cognitive Assessment score of 24 or below and the lower bound by a Mini Mental State Exam of 16 or greater); 4) be on stable doses of medications for motor (i.e. levodopa, dopaminergic agonist), cognitive (i.e. cholinesterase inhibitor), and psychiatric (i.e. antidepressant, antipsychotic) symptoms 3 months prior to the study; and 5) have a responsible caregiver at least 4 days/week. Potential participants will be excluded if they meet one of the following exclusion criteria: 1) have a clinically significant stroke or other neurological condition; 2) have other serious health underlying condition (e.g. cancer or unstable cardiac disease etc.).

Potential participants will be recruited from the Movement Disorders Clinics and Movement Disorders Centre at the London Health Sciences Centre, the Cognitive Neurology and Alzheimer's disease Research Centre and the Aging Brain and Memory Clinic both at Parkwood Institute (all located in London, ON, Canada), and from community Neurologists that routinely manage Parkinson's disease. Trial advertisement will be posted on strategic locations, shared in support groups, website, and social media via the Parkinson's Society South-Western Ontario as well as the online platform Fox Trials Finder. Individuals referred to the study by physicians and those who spontaneously contact the research team will be pre-screened via chart inspection and, if meet inclusion criteria, be offered a Screening visit.

All study visits will take place at Parkwood Institute. MRI scans will be performed at Robarts Research Institute and gait assessment at the Movement Disorders Centre at London Health Sciences Centre all located in London, ON, Canada.

\section{Screening visit}

In the screening visit, the inclusion and exclusion criteria will be reviewed followed by the performance of the Mini-Mental State Exam (MMSE) [31] and the Montreal Cognitive Assessment (MoCA) [32]. Then, participants' demographic information, medical history, and concomitant medication list will be recorded. A neurological and physical examination will be performed by a Neurologist (SHP). On a separate day, participants will have an MRI scan to rule out possible confounding neurological disease such as stroke or brain tumor and as a baseline for MRI-based biomarkers studies. Individuals with contra-indications to MRI or who choose not to have a MRI scan will have a CT scan. Individuals who pass the Screening visit will complete the Baseline assessment within 28 days.

\section{Randomization and blinding}

Randomization procedure will be completed by pharmacy staff using the website Research Randomizer (https://www.randomizer.org). Eligible participants will 


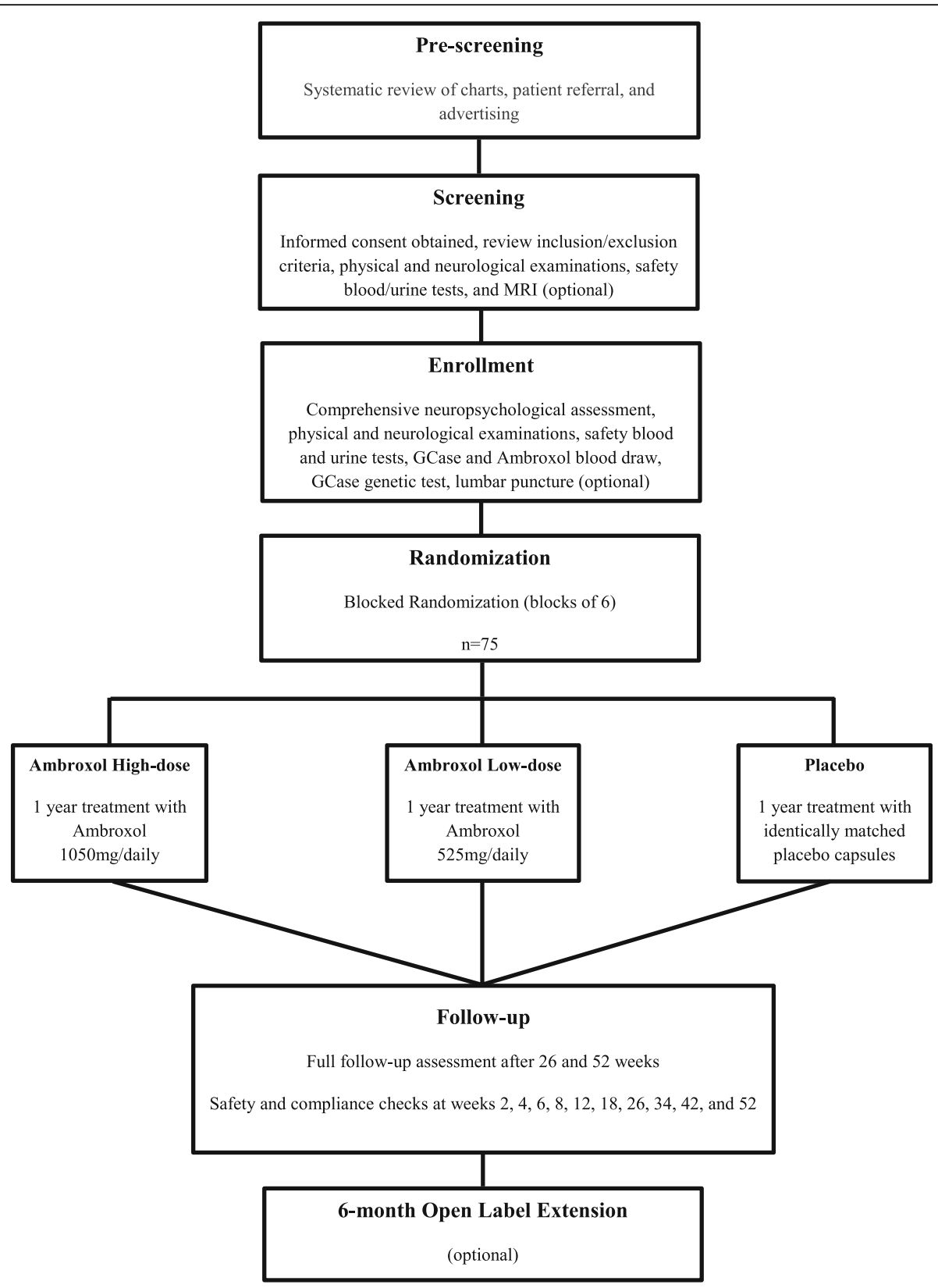

Fig. 1 Trial flow diagram

be randomly assigned into one of three arms: Ambroxol $1050 \mathrm{mg} /$ day (High-dose), Ambroxol $525 \mathrm{mg} /$ day (Low-dose) or placebo, in a 1:1:1 ratio in blocks of six participants each at baseline. Participants and all research personnel, with the exception of pharmacy staff, will be blinded to the randomization until the study is completed or a patient is purposely unblinded.

For blinding purposes, participants in all three arms will receive the same number of capsules per day. Medication will be blinded by over-encapsulating Ambroxol $75 \mathrm{mg}$ capsules (commercial product) and a matching placebo compound (microcrystalline cellulose) using opaque gelatin capsules (blue colour). Medications are packed as individual patient quantities consisting of one blister card for each week of treatment. The investigational product will be labeled by pharmacy in a double-blind manner (Ambroxol $75 \mathrm{mg} /$ placebo), with pre-assigned participant identification number.

\section{Intervention}

The treatment regimen consists of a titration phase and a maintenance phase. Participants begin the titration phase (weeks 1 and 2) taking 3 capsules a day $(225 \mathrm{mg}$ or $150 \mathrm{mg}$ or $0 \mathrm{mg}$ ) divided BID (2 capsules in the 
morning and 1 capsule in the evening). The first medication dose will be taken at the end of Baseline visit and in the presence of the study Neurologist. Possible side effects will be observed for $30 \mathrm{~min}$. Medication dose will increase every two weeks to 6 capsules on weeks 3 and 4, 10 capsules on weeks 5 and 6 , and 14 capsules on weeks 7 and 8 divided BID. At the end of titration (week 8) participants will have reached a maximum of $1050 \mathrm{mg}$ or $525 \mathrm{mg}$ or $0 \mathrm{mg}$ per day, depending on group allocation. In the maintenance phase, participants will remain in their maximum dose (1050 $\mathrm{mg}$ or $525 \mathrm{mg}$ or $0 \mathrm{mg}$ ) from week 9 to week 52 (End of Trial).

\section{Assessment procedures}

Participants will complete 11 visits to Parkwood Institute including baseline, weeks $2,4,6,8,12,18,26,34,42$, and 52 within one year of treatment. A comprehensive neuropsychological battery will be performed at baseline, week 26 , and week 52. The assessment of participants' motor and neuropsychiatric symptoms will also occur at these time-points. Physical examination will occur at all time-points but weeks 2 and 8 , whereas neurological examination will occur at baseline, weeks $6,12,18,34,42$, and 52 . Blood and urine samples as well as electrocardiograms will be collected at all visits. Cerebrospinal fluid will be collected through lumbar puncture at baseline, weeks 12, and 52 (optional). Finally, MRI scans will be collected at Screening and week 52 (optional). Table 1 illustrates all assessments performed at each time-point for the duration of the trial.

\section{Primary outcome measures}

The primary outcome measures will be the Alzheimer's disease Assessment Scale-cognitive subscale (ADAS-Cog) [33] and the Alzheimer's Disease Cooperative Study Clinician's Global Impression of Change (ADCS-CGIC) [34]. The ADAS-Cog is a comprehensive assessment battery composed by 11 items (word-recall, commands, constructional praxis, naming, ideational praxis, orientation, word recognition, remembering instructions, comprehension, word finding, and spoken language). The test is rated on a scale from 0 to 70 , where greater score represents greater impairment. Although designed for Alzheimer's disease where it is considered a gold standard, the ADAS-Cog has been used effectively in many clinical trials of PDD including large randomized trials [33, 35-38].

The Alzheimer's Disease Cooperative Study Clinician's Global Impression of Change (ADCS-CGIC) scale [34] is a 7-point scale for rating patient function in cognition, behavior, and activities of daily living. ADCS-CGIC is a standard test in clinical trials in Alzheimer's disease and has been useful in trials with PDD [37-39]. This scale was also included because the FDA requires measures of global function in cognitive clinical trials [40]. Thus, this measure will provide critical evidence for potential multi-centre trials in the future. At baseline, information regarding mental/cognitive state (arousal/attention, orientation, memory, language, praxis, and judgment/problem solving), behaviour (thought content, hallucinations/delusions, behaviour/ mood, sleep and appetite, and neurological/psychomotor), and functioning (instrumental functional ability and social function) are collected from both patient and caregiver. This information will be used as a reference for the assessment of change throughout the study. In this clinical trial, the individual performing the ADCS-CIGC will be an independent rater blinded to participant's progression in the trial (i.e. group allocation, compliance, adverse events, and performance in other outcome measures).

\section{Secondary outcome measures \\ Neuropsychological tests}

Secondary cognitive measures include the Clinical Dementia Rating Scale [41], Parkinson's disease Cognitive Rating Scale [42], Trail Making Test [43], and Stroop test [44].

\section{Motor function}

The assessment of motor function will be completed using the Movement Disorders Society Unified Parkinson's Disease Rating Scale (MDS-UPDRS) [45], Purdue Pegboard [46], Timed Up and Go [47], and gait kinematics.

\section{Mood and neuropsychiatric symptoms}

Neuropsychiatric symptoms will be assessed with the Neuropsychiatric Inventory [48] and Geriatric Depression Scale [49].

\section{CSF and brain imaging biomarkers}

CSF collected through lumbar puncture (levels of $\alpha$-synuclein, Tau, phospho-Tau and beta amyloid-42) and brain imaging (brain ventricle and hippocampal volume, and metabolic indicators of neurodegeneration) biomarkers will be collected.

\section{Pharmacokinetics and pharmacodynamics}

Pharmacokinetics and pharmacodynamics of Ambroxol will be examined through blood concentrations during a dose titration phase and measurements of GCase activity in lymphocytes.

\section{Genetics}

The GBA1 gene will be sequenced in all participants to identify carriers and non-carriers of mutations and any other allelic variations in the gene for $\beta$-Glucocerebrosidase (GCase; gene name GBA1).

\section{Safety}

Routine blood draws (chemistry, hematology, and coagulation) and urine chemistry will be collected in all visits, and any abnormal results will be shared with each participant's 
Table 1 List of assessments performed in each visit

\begin{tabular}{|c|c|c|c|c|c|c|c|c|c|c|c|c|}
\hline Visit Name & Screen & Baseline & Week 2 & Week 4 & Week 6 & Week 8 & Week 12 & Week 18 & Week 26 & Week 34 & Week 42 & Week 52 \\
\hline Consent & $x$ & & & & & & & & & & & \\
\hline \multicolumn{13}{|l|}{ Medical History } \\
\hline Medical History & $x$ & & & & & & & & & & & \\
\hline Demographics & $x$ & & & & & & & & & & & \\
\hline $\begin{array}{l}\text { Review inclusion and } \\
\text { exclusion criteria }\end{array}$ & $x$ & $x$ & & & & & & & & & & \\
\hline MRI & $x$ & & & & & & & & & & & $x$ \\
\hline Randomization & & $x$ & & & & & & & & & & \\
\hline \multicolumn{13}{|l|}{ Medication } \\
\hline In clinic first dose & & $x$ & & & & & & & & & & \\
\hline Compliance assessment & & & $x$ & $x$ & $x$ & $x$ & $x$ & $x$ & $x$ & $x$ & $x$ & $x$ \\
\hline $\begin{array}{l}\text { Concomitant Medication } \\
\text { Check }\end{array}$ & $x$ & $x$ & $x$ & $x$ & $x$ & $x$ & $x$ & $x$ & $x$ & $x$ & $x$ & $x$ \\
\hline \multicolumn{13}{|l|}{ Safety Assessments } \\
\hline Adverse Event Review & & $x$ & $x$ & $x$ & $x$ & $x$ & $x$ & $x$ & $x$ & $x$ & $x$ & $x$ \\
\hline Interview & $x$ & $x$ & $x$ & $x$ & $x$ & $x$ & $x$ & $x$ & $x$ & $x$ & $x$ & $x$ \\
\hline Chemistry Blood Draw & $x$ & $x$ & $x$ & $x$ & $x$ & $x$ & $x$ & $x$ & $x$ & $x$ & $x$ & $x$ \\
\hline Hematology Blood Draw & $x$ & $x$ & $x$ & $x$ & $x$ & $x$ & $x$ & $x$ & $x$ & $x$ & $x$ & $x$ \\
\hline Coagulation Blood Draw & $x$ & $x$ & $x$ & $x$ & $x$ & $x$ & $x$ & $x$ & $x$ & $x$ & $x$ & $x$ \\
\hline Genetics Blood Draw & & $x$ & & & & & & & & & & \\
\hline GCase Blood Draw & & $x$ & $x$ & $x$ & $x$ & $x$ & $x$ & & $x$ & & & $x$ \\
\hline Ambroxol Blood Draw & & $x$ & $x$ & $x$ & $x$ & $x$ & $x$ & & $x$ & & & $x$ \\
\hline Urinalysis & $x$ & $x$ & $x$ & $x$ & $x$ & $x$ & $x$ & $x$ & $x$ & $x$ & $x$ & $x$ \\
\hline Standing Blood Pressure & $x$ & $x$ & $x$ & $x$ & $x$ & $x$ & $x$ & $x$ & $x$ & $x$ & $x$ & $x$ \\
\hline Seating Blood Pressure & $x$ & $x$ & $x$ & $x$ & $x$ & $x$ & $x$ & $x$ & $x$ & $x$ & $x$ & $x$ \\
\hline Height & $x$ & & & & & & & & & & & \\
\hline Weight & $x$ & $x$ & $x$ & $x$ & $x$ & $x$ & $x$ & $x$ & $x$ & $x$ & $x$ & $x$ \\
\hline ECG & $x$ & $x$ & $x$ & $x$ & $x$ & $x$ & $x$ & $x$ & $x$ & $x$ & $x$ & $x$ \\
\hline Physical Exam & $x$ & $x$ & & $x$ & $x$ & & $x$ & $x$ & $x$ & $x$ & $x$ & $x$ \\
\hline Neurological Exam & $x$ & $x$ & & & $x$ & & $x$ & $x$ & $x$ & $x$ & $x$ & $x$ \\
\hline CSF GCase & & $x$ & & & & & $x$ & & & & & $x$ \\
\hline CSF Ambroxol & & $x$ & & & & & $x$ & & & & & $x$ \\
\hline CSF Safety & & $x$ & & & & & $x$ & & & & & $x$ \\
\hline MMSE & $x$ & $x$ & & $x$ & $x$ & & $x$ & $x$ & $x$ & $x$ & $x$ & $x$ \\
\hline Gait Kinematics & & $x$ & & & & & & & $x$ & & & $x$ \\
\hline Timed up and Go & & $x$ & & & & & & & $x$ & & & $x$ \\
\hline Purdue Pegboard & & $x$ & & & & & & & $x$ & & & $x$ \\
\hline UPDRS & & $x$ & & & & & & & $x$ & & & $x$ \\
\hline \multicolumn{13}{|l|}{ Cognitive Assessments } \\
\hline ADAS-Cog & & $x$ & & & & & & & $x$ & & & $x$ \\
\hline CGIC & & $x$ & & & & & & & $x$ & & & $x$ \\
\hline PD-CRS & & $x$ & & & & & & & $x$ & & & $x$ \\
\hline NPI & & $x$ & & & & & & & $x$ & & & $x$ \\
\hline CDR & & $x$ & & & & & & & $x$ & & & $x$ \\
\hline MoCA & & $x$ & & & & & & & $x$ & & & $x$ \\
\hline
\end{tabular}


Table 1 List of assessments performed in each visit (Continued)

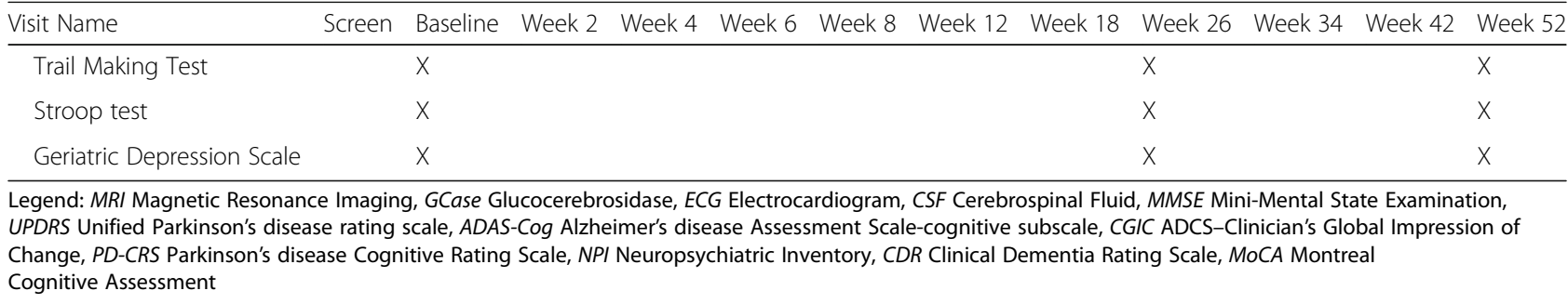

family physician (primary care) with each participant's permission. Results from laboratory analyses will be reviewed by the study's principal investigator and periodically by an independent data and safety monitoring board (DSMB).

Each participant will be asked at every visit whether or not any adverse event occurred. Data on adverse events will be reviewed by the study's principal investigator, recorded in participants' files, and presented to the DSMB at meetings that occur every 6 months. Serious adverse events (death, hospitalization, or leading to disability/incapacity) will be reported to both Western University research ethics board as well as Health Canada.

Ambroxol is generally well tolerated [50]. The European Core Safety Profile lists headache, dizziness, tachycardia, flushing, nausea, vomiting, diarrhea and abdominal pain as side effects [51]. Recently severe skin lesions such as Stevens-Johnson Syndrome and toxic epidermal necrolysis (TEN) was added as a warning, but it appears to be so rare that it is not possible to determine an event rate. We were unable to identify any literature or case reports of severe reactions. A recent Post Marketing survey of 2707 individuals taking Ambroxol syrup found the $2.5 \%$ of patients reported adverse events, which were mostly mild, mainly GI (abdominal pain, diarrhea, nausea, vomiting). There were no serious adverse events.

In the event of minor adverse events (e.g. gastrointestinal side effects), patients will be allowed to spread their dose over a longer interval, or to reduce their dose to the highest dose tolerated. The dose can be advanced again after 2 weeks. For significant but non-threatening laboratory abnormalities, doses can be reduced to the highest dose tolerated. Participants will be allowed to stop taking medication if there is concern about a drug reaction of a clinically significant alteration in laboratory values. They can be re-challenged 2-4 weeks after these abnormalities have resolved.

In order to confirm participant self-reported adherence to the study medication, the number of pills returned in each visit will be counted and recorded. Unused pills will be returned to pharmacy for destruction.

\section{Sample size}

Because Ambroxol has never been used for this indication, there is insufficient information available to perform a sample size calculation. However, it is known that individuals with Alzheimer's disease typically have 6-7 points change in the ADAS-Cog in 1 year [52] and there are data suggesting that PDD patients may decline in a similar fashion [53-56]. The required sample size was calculated to ensure power of at least $80 \%$ in detecting two a priori comparisons, i.e., 1) the difference in average change in the ADAS-Cog scores between patients receiving Ambroxol and placebo, and 2) the difference in the ADAS-Cog scores between low dose Ambroxol and high dose Ambroxol, using 2-sided tests at the 5\% significance level. The calculation assumed that the one-year change scores from baseline in ADAS-Cog scores are 7 points, 5 points, and 0 points for placebo, low dose, and high dose groups, respectively, with a common standard deviation of 5.5 points. Using the procedure 'SAS proc glmpower' (from SAS version 9.3, SAS Institute Inc., Cary, NC, USA), a total of 60 patients (20 per group) will provide $83.6 \%$ power to detect a difference of 2.5 between the average of two Ambroxol groups and placebo, and $80.7 \%$ power to a difference of 5 points between the low and high doses of Ambroxol. To account for attrition, 5 additional patients per group will be enrolled in the study.

\section{Statistical analysis}

Descriptive statistics such as mean, standard deviation, and frequency by treatment arm will be used to summarize patient baseline characteristics.

The ADAS-Cog data will be analyzed using a restricted maximum likelihood-based repeated measures approach. The analyses will include the fixed, categorical effects of treatment, visit time, and the continuous, fixed covariate of baseline ADAS-Cog score, and interaction between treatment and visit. An unstructured (co)variance structure shared across treatment groups will be used to model within-patient errors. The Kenward-Roger approximation will be used to estimate denominator degrees of freedom and adjust standard errors. The primary analysis will be the contrast at week 52, comparing i) the average of the two Ambroxol groups versus placebo; ii) low-dose Ambroxol group versus high-dose Ambroxol group. All tests will be carried out at 2-sided 5\% significance level.

Similar approach will be adopted for ADCS-CGIC and the secondary outcomes that are continuous. Binary outcomes will be analyzed using logistic regression analysis. 
All analyses will be done using SAS version 9.3 (SAS Institute Inc., Cary, NC, USA).

\section{Discussion}

Cognitive decline and its implications to the life of individuals with PDD represent a significant burden to patients, caregivers, and the health care system. Current pharmacological therapies are helpful for cognitive symptoms in PDD but are unable to modify the course of disease progression, making treatment for cognition in PDD a major unmet need. Increasing levels of the GCase enzyme has been proposed as a potential therapy for slowing neurodegeneration in Parkinson's disease, and consequently PDD. There is increasing evidence in cell culture and animal models of PD that Ambroxol can raise levels of GCase. Strikingly, increased levels of GCase were accompanied by decreased levels of $\alpha$-synuclein aggregation in brain areas of mice treated with Ambroxol. The present trial is the first to test Ambroxol in human subjects with PDD. The effects of Ambroxol will be measured using standardized cognitive, functional, behavioural, biochemical, as well as brain imaging outcomes. Results from this clinical trial may have a large impact in the research of disease-modifying therapies for PDD and other synucleinopathies including Parkinson's disease and Lewy Body dementia. Importantly, Ambroxol is a drug already commercially available in most of the world and has an excellent safety record. Thus, repurposing Ambroxol for the treatment of PDD may expedite a potential disease-modifying treatment and circumvent a lengthy drug development process.

\section{Abbreviations}

ADAS-Cog: Alzheimer's disease assessment scale-cognitive subscale; ADCSCGIC: Alzheimer's disease cooperative study clinician's global impression of change; CSF: Cerebrospinal fluid; DSMB: Data and safety monitoring board; GBA1: Gene encoding the protein $\beta$-Glucocerebrosidase; GCase: $\beta$ -

Glucocerebrosidase; iPSC: induced pluripotent stem cell; MMSE: Mini-mental state exam; MoCA: Montreal cognitive assessment; MRI: Magnetic resonance imaging; PDD: Parkinson's disease dementia; TEN: toxic epidermal necrolysis

\section{Acknowledgements \\ The authors would like to thank Dr. Richard Camicioli and Dr. Mario Masellis for advice on the diagnosis and assessment of PDD, and on clinical trial design. In addition, we would like to thank Greydon Gilmore, Patricia Sargeant, Abigail Korczak, and Elsa Mann for their contribution to data collection, Dr. Daniel Mendonca and Dr. Dwight Stewart for their contribution to participants' recruitment, and Dr. Geoffrey Pickering, Dr. Sachin Pandy, Dr. Manas Sharma, and Dr. Michael Mayich for aiding in the clinical assessment of patients.}

\section{Funding}

The present study is funded by the Weston Brain Institute CT140053. The Weston Brain Institute provided peer review of the trial as part of the grant funding process, which included an expert advice on Parkinson's disease clinical trial design.

Availability of data and materials Not applicable.

\section{Authors' contributions}

SHP, EF, RB, SAM, JW, MB, RGT, CAR, GZ, RAH, DM, PM, MEJ, MJ, JM, KC, CRAS, and ZL made substantial contributions to conception, design, data acquisition and analysis in this trial. CRAS, JM, KC, and SHP drafted the manuscript and revised it critically for important intellectual content. SHP, EF, RB, SAM, JW, MB, RGT, CAR, GZ, RAH, DM, PM, MEJ, MJ, JM, KC, CRAS, and ZL have given final approval of the version to be published and agreed to be accountable for all aspects of the work in ensuring that questions related to the accuracy or integrity of any part of the work are appropriately investigated and resolved. All authors read and approved the final manuscript.

\section{Ethics approval and consent to participate}

The present study has been approved by Western University Health Science Research Ethics Board (Protocol ID: 105234) and all individuals will sign a consent form prior to participation. The study coordinator will review the letter of information, answer questions, and obtain consent from participant and caregiver/next of kin or power of attorney (POA) at the screening visit. Because the ability to consent can be difficult to assess and can change over time, written consent will be obtained from both participants with dementia and caregiver/next of kin or POA to ensure the rights of participants who may have deficits in comprehension or decision making.

\section{Consent for publication}

Not applicable.

\section{Competing interests}

The authors declare that they have no competing interests.

\section{Publisher's Note}

Springer Nature remains neutral with regard to jurisdictional claims in published maps and institutional affiliations.

\section{Author details}

${ }^{1}$ Cognitive Neurology and Alzheimer's Disease Research Centre, Parkwood Institute - Main Building, Room A230, 550, Wellington Road, London, Ontario N6G OA7, Canada. 'Lawson Health Research Institute, London, Ontario, Canada. ${ }^{3}$ Deparment of Clinical Neurological Science, Schulich School of Medicine and Dentistry, Western University, London, Ontario, Canada. ${ }^{4}$ Division of Geriatric Medicine, Schulich School of Medicine and Dentistry, Western University, London, Ontario, Canada. ${ }^{5}$ Department of Biochemistry, Schulich School of Medicine and Dentistry, Western University, London, Ontario, Canada. ${ }^{6}$ Department of Medicine, Schulich School of Medicine and Dentistry, Western University, London, Ontario, Canada. ${ }^{7}$ Department of Physiology and Pharmacology, Schulich School of Medicine and Dentistry, Western University, London, Ontario, Canada. ${ }^{8}$ Department of Medical Biophysics, Schulich School of Medicine and Dentistry, Western University, London, Ontario, Canada. ${ }^{9}$ Department of Epidemiology and Biostatistics, Schulich School of Medicine and Dentistry, Western University, London, Ontario, Canada. ${ }^{10}$ Laboratory of Medicine and Pathobiology, The Hospital for Sick Children, Toronto, Ontario, Canada. ${ }^{11}$ Robarts Research Institute, Western University, London, Ontario, Canada.

Received: 4 September 2018 Accepted: 1 February 2019

Published online: 09 February 2019

\section{References}

1. Moore SF, Barker RA. Predictors of Parkinson's disease dementia: towards targeted therapies for a heterogeneous disease. Parkinsonism Relat Disord. 2014;20(Suppl 1):S104-7.

2. Braak H, Ghebremedhin E, Rub U, Bratzke H, Del Tredici K. Stages in the development of Parkinson's disease-related pathology. Cell Tissue Res. 2004; 318(1):121-34

3. Halliday GM, Leverenz JB, Schneider JS, Adler CH. The neurobiological basis of cognitive impairment in Parkinson's disease. Mov Disord. 2014;29(5):634-50.

4. Williams-Gray CH, Mason SL, Evans JR, Foltynie T, Brayne C, Robbins TW, et al. The CamPalGN study of Parkinson's disease: 10-year outlook in an incident population-based cohort. J Neurol Neurosurg Psychiatry. 2013; 84(11):1258-64.

5. Barone P, Antonini A, Colosimo C, Marconi R, Morgante L, Avarello TP, et al. The PRIAMO study: a multicenter assessment of nonmotor symptoms and 
their impact on quality of life in Parkinson's disease. Mov Disord. 2009; 24(11):1641-9.

6. Aarsland D, Larsen JP, Tandberg E, Laake K. Predictors of nursing home placement in Parkinson's disease: a population-based, prospective study. J Am Geriatr Soc. 2000;48(8):938-42.

7. Buter TC, van den Hout A, Matthews FE, Larsen JP, Brayne C, Aarsland D. Dementia and survival in Parkinson disease: a 12-year population study. Neurology. 2008;70(13):1017-22.

8. Deane KH, Flaherty H, Daley DJ, Pascoe R, Penhale B, Clarke CE, et al. Priority setting partnership to identify the top 10 research priorities for the management of Parkinson's disease. BMJ Open. 2014;4(12):e006434.

9. Emre M, Ford PJ, Bilgic B, Uc EY. Cognitive impairment and dementia in Parkinson's disease: practical issues and management. Mov Disord. 2014 29(5):663-72.

10. Sidransky E, Lopez $\mathrm{G}$. The link between the GBA gene and parkinsonism. Lancet Neurol. 2012;11(11):986-98.

11. Balducci C, Pierguidi L, Persichetti E, Parnetti L, Sbaragli M, Tassi C, et al. Lysosomal hydrolases in cerebrospinal fluid from subjects with Parkinson's disease. Mov Disord. 2007;22(10):1481-4.

12. Gegg ME, Burke D, Heales SJ, Cooper JM, Hardy J, Wood NW, et al. Glucocerebrosidase deficiency in substantia nigra of parkinson disease brains. Ann Neurol. 2012;72(3):455-63.

13. Murphy KE, Gysbers AM, Abbott SK, Tayebi N, Kim WS, Sidransky E, et al. Reduced glucocerebrosidase is associated with increased alpha-synuclein in sporadic Parkinson's disease. Brain. 2014;137(Pt 3):834-48.

14. Mazzulli JR, Xu YH, Sun Y, Knight AL, McLean PJ, Caldwell GA, et al. Gaucher disease glucocerebrosidase and alpha-synuclein form a bidirectional pathogenic loop in synucleinopathies. Cell. 2011;146(1):37-52.

15. Sardi SP, Clarke J, Kinnecom C, Tamsett TJ, Li L, Stanek LM, et al. CNS expression of glucocerebrosidase corrects alpha-synuclein pathology and memory in a mouse model of Gaucher-related synucleinopathy. Proc Natl Acad Sci U S A. 2011;108(29):12101-6.

16. Xu YH, Sun Y, Ran H, Quinn B, Witte D, Grabowski GA. Accumulation and distribution of alpha-synuclein and ubiquitin in the CNS of Gaucher disease mouse models. Mol Genet Metab. 2011;102(4):436-47.

17. Manning-Bog AB, Schule B, Langston JW. Alpha-synucleinglucocerebrosidase interactions in pharmacological Gaucher models: a biological link between Gaucher disease and parkinsonism. Neurotoxicology. 2009;30(6):1127-32.

18. Sardi SP, Clarke J, Viel C, Chan M, Tamsett TJ, Treleaven CM, et al. Augmenting CNS glucocerebrosidase activity as a therapeutic strategy for parkinsonism and other Gaucher-related synucleinopathies. Proc Natl Acad Sci U S A. 2013;110(9):3537-42.

19. Malerba M, Ragnoli B. Ambroxol in the 21st century: pharmacological and clinical update. Expert Opin Drug Metab Toxicol. 2008;4(8):1119-29.

20. Maegawa GH, Tropak MB, Buttner JD, Rigat BA, Fuller M, Pandit D, et al. Identification and characterization of ambroxol as an enzyme enhancement agent for Gaucher disease. J Biol Chem. 2009;284(35):23502-16.

21. Morello JP, Petaja-Repo UE, Bichet DG, Bouvier M. Pharmacological chaperones: a new twist on receptor folding. Trends Pharmacol Sci. 2000; 21(12):466-9.

22. Parenti G, Andria G, Valenzano KJ. Pharmacological chaperone therapy: preclinical development, clinical translation, and prospects for the treatment of lysosomal storage disorders. Mol Ther. 2015;23(7):1138-48.

23. Muntau AC, Leandro J, Staudigl M, Mayer F, Gersting SW. Innovative strategies to treat protein misfolding in inborn errors of metabolism: pharmacological chaperones and proteostasis regulators. J Inherit Metab Dis. 2014;37(4):505-23.

24. Luan Z, Li L, Higaki K, Nanba E, Suzuki Y, Ohno K. The chaperone activity and toxicity of ambroxol on Gaucher cells and normal mice. Brain and Development. 2013;35(4):317-22.

25. McNeill A, Magalhaes J, Shen C, Chau KY, Hughes D, Mehta A, et al. Ambroxol improves lysosomal biochemistry in glucocerebrosidase mutation-linked Parkinson disease cells. Brain. 2014;137(Pt 5):1481-95.

26. Yang SY, Beavan M, Chau KY, Taanman JW, Schapira AHV. A human neural crest stem cell-derived dopaminergic neuronal model recapitulates biochemical abnormalities in GBA1 mutation carriers. Stem Cell Reports. 2017;8(3):728-42.

27. Migdalska-Richards A, Daly L, Bezard E, Schapira AH. Ambroxol effects in glucocerebrosidase and alpha-synuclein transgenic mice. Ann Neurol. 2016; 80(5):766-75.
28. Migdalska-Richards A, Ko WKD, Li Q, Bezard E, Schapira AHV. Oral ambroxol increases brain glucocerebrosidase activity in a nonhuman primate. Synapse. 2017;71(7).

29. Narita A, Shirai K, Itamura S, Matsuda A, Ishihara A, Matsushita K, et al. Ambroxol chaperone therapy for neuronopathic Gaucher disease: a pilot study. Ann Clin Transl Neurol. 2016;3(3):200-15.

30. Zimran A, Altarescu G, Elstein D. Pilot study using ambroxol as a pharmacological chaperone in type 1 Gaucher disease. Blood Cells Mol Dis. 2013;50(2):134-7.

31. Folstein MF, Folstein SE, McHugh PR. "mini-mental state". A practical method for grading the cognitive state of patients for the clinician. J Psychiatr Res. 1975;12(3):189-98.

32. Nasreddine ZS, Phillips NA, Bedirian V, Charbonneau S, Whitehead V, Collin I, et al. The Montreal cognitive assessment, MoCA: a brief screening tool for mild cognitive impairment. J Am Geriatr Soc. 2005:53(4):695-9.

33. Rosen WG, Mohs RC, Davis KL. A new rating scale for Alzheimer's disease. Am J Psychiatry. 1984;141(11):1356-64.

34. Schneider LS, Olin JT, Doody RS, Clark CM, Morris JC, Reisberg B, et al. Validity and reliability of the Alzheimer's Disease Cooperative Study-Clinical Global Impression of Change. The Alzheimer's Disease Cooperative Study. Alzheimer Dis Assoc Disord. 1997:11(Suppl 2):S22-32.

35. Burn D, Emre M, McKeith I, De Deyn PP, Aarsland D, Hsu C, et al. Effects of rivastigmine in patients with and without visual hallucinations in dementia associated with Parkinson's disease. Mov Disord. 2006;21(11):1899-907.

36. Dubois B, Tolosa E, Katzenschlager R, Emre M, Lees AJ, Schumann G, et al. Donepezil in Parkinson's disease dementia: a randomized, double-blind efficacy and safety study. Mov Disord. 2012;27(10):1230-8.

37. Emre M, Aarsland D, Albanese A, Byrne EJ, Deuschl G, De Deyn PP, et al Rivastigmine for dementia associated with Parkinson's disease. N Engl J Med. 2004:351(24):2509-18.

38. Ravina B, Putt M, Siderowf A, Farrar JT, Gillespie M, Crawley A, et al. Donepezil for dementia in Parkinson's disease: a randomised, double blind, placebo controlled, crossover study. J Neurol Neurosurg Psychiatry. 2005; 76(7):934-9.

39. Aarsland D, Laake K, Larsen JP, Janvin C. Donepezil for cognitive impairment in Parkinson's disease: a randomised controlled study. J Neurol Neurosurg Psychiatry. 2002;72(6):708-12.

40. McLendon BM, Doraiswamy PM. Defining meaningful change in Alzheimer's disease trials: the donepezil experience. J Geriatr Psychiatry Neurol. 1999; 12(1):39-48.

41. Morris JC. The clinical dementia rating (CDR): current version and scoring rules. Neurology. 1993;43(11):2412-4.

42. Pagonabarraga J, Kulisevsky J, Llebaria G, Garcia-Sanchez C, Pascual-Sedano B, Gironell A. Parkinson's disease-cognitive rating scale: a new cognitive scale specific for Parkinson's disease. Mov Disord. 2008;23(7):998-1005.

43. Tombaugh TN. Trail making test a and B: normative data stratified by age and education. Arch Clin Neuropsychol. 2004;19(2):203-14.

44. Stroop JR. Studies of interference in serial verbal reactions [Ph D]. Nashville, Tenn: George Peabody College for Teachers; 1935.

45. Goetz CG, Tilley BC, Shaftman SR, Stebbins GT, Fahn S, Martinez-Martin P, et al. Movement Disorder Society-sponsored revision of the unified Parkinson's disease rating scale (MDS-UPDRS): scale presentation and clinimetric testing results. Mov Disord. 2008;23(15):2129-70.

46. Tiffin J, Asher EJ. The Purdue pegboard; norms and studies of reliability and validity. J Appl Psychol. 1948;32(3):234-47.

47. Podsiadlo D, Richardson S. The timed "up \& go": a test of basic functional mobility for frail elderly persons. J Am Geriatr Soc. 1991;39(2):142-8.

48. Cummings JL, Mega M, Gray K, Rosenberg-Thompson S, Carusi DA, Gornbein J. The neuropsychiatric inventory: comprehensive assessment of psychopathology in dementia. Neurology. 1994;44(12):2308-14.

49. Yesavage JA, Brink TL, Rose TL, Lum O, Huang V, Adey M, et al. Development and validation of a geriatric depression screening scale: a preliminary report. J Psychiatr Res. 1982;17(1):37-49.

50. Schulz M, Hammerlein A, Hinkel U, Weis G, Gillissen A. Safety and usage pattern of an over-the-counter ambroxol cough syrup: a community pharmacy-based cohort study. Int J Clin Pharmacol Ther. 2006:44(9):409-21.

51. Core Safety Profile. In: Ambroxol. Federal Institute for Drugs and Medical Devices. 2010. https://www.bfarm.de/SharedDocs/Downloads/EN/Drugs/ vigilance/PSURs/csp/a-b/ambroxol.pdf?_blob=publicationFile\&v=3

52. Schneider LS. Treatment of Alzheimer's disease with cholinesterase inhibitors. Clin Geriatr Med. 2001;17(2):337-58. 
53. Poewe W, Wolters E, Emre M, Onofrj M, Hsu C, Tekin S, et al. Long-term benefits of rivastigmine in dementia associated with Parkinson's disease: an active treatment extension study. Mov Disord. 2006;21 (4):456-61.

54. Marras C, McDermott MP, Rochon PA, Tanner CM, Naglie G, Lang AE, et al. Predictors of deterioration in health-related quality of life in Parkinson's disease: results from the DATATOP trial. Mov Disord. 2008;23(5):653-9 quiz 776.

55. Olanow CW, Rascol O, Hauser R, Feigin PD, Jankovic J, Lang A, et al. A double-blind, delayed-start trial of rasagiline in Parkinson's disease. N Engl J Med. 2009;361(13):1268-78

56. Rascol O, Fitzer-Attas CJ, Hauser R, Jankovic J, Lang A, Langston JW, et al. A double-blind, delayed-start trial of rasagiline in Parkinson's disease (the ADAGIO study): prespecified and post-hoc analyses of the need for additional therapies, changes in UPDRS scores, and non-motor outcomes. Lancet Neurol. 2011;10(5):415-23.

Ready to submit your research? Choose BMC and benefit from:

- fast, convenient online submission

- thorough peer review by experienced researchers in your field

- rapid publication on acceptance

- support for research data, including large and complex data types

- gold Open Access which fosters wider collaboration and increased citations

- maximum visibility for your research: over $100 \mathrm{M}$ website views per year

At BMC, research is always in progress.

Learn more biomedcentral.com/submissions 